\title{
Creating the Future of Physical Activity Surveillance in the United States: Better Data for Better Health
}

\author{
James F. Sallis and Russell R. Pate
}

An extensive body of knowledge documents the powerful influence that physical activity exerts on the public's health. ${ }^{1-4}$ This body of knowledge has grown dramatically over the past halfcentury, and it is now well-established that physical activity produces a wide range of health benefits in essentially all segments of the population. ${ }^{1,3,5-9}$ Accordingly, over the past several decades, public health organizations across the world have increasingly recognized the importance of physical activity. ${ }^{10,11}$ In the United States, this expanding public health role is perhaps best exemplified by the Physical Activity Guidelines for Americans, which were first released in 2008 and then updated in 2018. ${ }^{12,13}$ These guidelines include detailed physical activity recommendations for population subgroups ranging from preschoolers to older persons.

Most Americans do not fully meet the federal physical activity guidelines ${ }^{14}$ and, consequently, well-resourced public health initiatives are needed to increase physical activity in the US population. Surveillance is an essential component of public health planning and practice, as it provides ongoing, systematic collection, analysis, and interpretation of outcome-specific data for use in planning, implementation, and evaluation of public health activities. As public health agencies have recognized the importance of physical activity, they have included physical activity monitoring in public health surveillance systems. In contrast to other health behaviors, such as diet and tobacco use, physical activity is a relatively recent focus of public health, and many gaps still exist in surveillance of physical activity. This commentary makes the case for why and how to strengthen physical activity surveillance in the United States. The specific purposes of the commentary are to (1) highlight the strengths and limitations of current physical activity surveillance in the United States, (2) summarize recommended actions for enhancement of physical activity surveillance, and (3) call for actions across multiple societal sectors to support creation of robust physical activity surveillance in the United States.

\section{Existing Resources and Strengths}

As public health agencies have recognized the importance of promoting physical activity, prominent public health surveillance systems have increasingly included surveillance of physical activity. These include the Behavioral Risk Factor Surveillance System $(\mathrm{BRFSS})^{15}$ and the Youth Risk Behavior Survey (YRBS), ${ }^{16}$ which regularly assess person-level physical activity behaviors in US adults and high school students, respectively. Both of these systems include self-report methods for assessing compliance with physical

Sallis is with the Herbert Wertheim School of Public Health, University of California, San Diego, CA, USA. Pate is with the Department of Exercise Science, University of South Carolina, Columbia, SC, USA. Pate (rpate@ mailbox.sc.edu) is corresponding author. activity guidelines. ${ }^{13}$ The National Health and Nutrition Examination Survey (NHANES) ${ }^{17}$ has assessed person-level physical activity behavior across a wide age range, using both self-report and device-based methods during some survey cycles. The National Health Interview Survey ${ }^{18}$ assesses physical activity behavior in adults. So, an established strength of physical activity surveillance in the United States is that key systems currently assess self-reported compliance with physical activity guidelines in adults and high school students.

Some surveillance systems have included items that assessed person-level participation in specific forms of physical activity. Items to assess specific forms of physical activity were included in the nonrecurring National Youth Fitness Survey ${ }^{19}$ and to a limited extent in the American Time Use Survey, ${ }^{20}$ NHANES, BRFSS, and YRBS. Transportation-related physical activities are monitored in the National Household Travel Survey. ${ }^{21}$

Policies and programs that support physical activity are monitored to a limited extent. The School Health Policies and Programs Survey provided a comprehensive assessment of school-based initiatives to promote physical activity in students, but that survey has been terminated.22 Classification of Laws Associated with Students has included assessment of state laws related to school physical education. ${ }^{23}$

\section{Recommended Enhancements to Physical Activity Surveillance in the United States}

The 2019 National Academies of Science, Engineering, and Medicine (NASEM) Consensus Report on Implementing Strategies to Enhance Physical Activity Surveillance in the United States ${ }^{24}$ describes a plan for making physical activity surveillance more effective. The NASEM report built on earlier documents that identified goals and strategies for the next generation of physical activity surveillance ${ }^{25}$ and a 2018 report that proposed specific actions to achieve those goals. ${ }^{26}$ The Consensus Report focused on steps needed to implement actions to expand physical activity surveillance in 4 areas-children, health care, workplaces, and community supports for physical activity - and included 22 strategies and 59 specific actions. Table 1 lists the 22 surveillance strategies and estimated level of costs of implementing each strategy, as discussed in a commentary in the American Journal of Public Health. ${ }^{27}$

As noted above, most of the current physical activity surveillance systems assess person-level physical activity in various populations. Although these systems have provided valuable long-term data on behavior at the population level, they do not provide evidence that can be used to tailor interventions to specific subgroups or track trends in the conditions that influence physical activity. Thus, the NASEM report ${ }^{24}$ proposed an expanded vision and new goals for physical activity surveillance for the purpose of 


\section{Table 1 Recommended Strategies to Enhance Physical Activity Surveillance and Estimated Relative Costs for Implementation}

\section{Children}

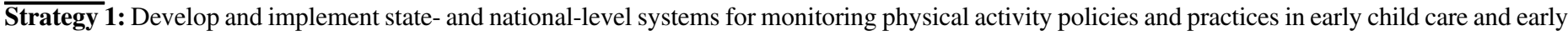
childhood education settings. Estimated costs: high.

Strategy 2: Enhance existing surveillance systems for monitoring elementary through high school-based physical activity policies and programs. Estimated costs: medium.

Strategy 3: Develop a protocol that leverages ongoing administration of physical fitness tests, such as FitnessGram, for the purpose of monitoring fitness levels of children and youth. Estimated costs: low.

Strategy 4: Expand objective monitoring of physical activity in children (ages 3-18 y) by incorporating validated wearable technologies into existing surveillance systems. Estimated costs: medium.

Strategy 5: Develop a system for monitoring community-level availability of organized sports and other physical activity programs for children. Estimated costs: medium.

Strategy 6: Identify features of the built environment that are most likely to influence physical activity in children, and embed an assessment of the perceived availability and use of these features into existing surveillance systems. Estimated costs: low.

\section{Health care}

Strategy 7: Develop surveillance systems to monitor the prevalence of physical activity assessment in adults through expanded integration of a standard physical activity vital sign in health care delivery. Estimated costs: medium.

Strategy 8: Develop surveillance systems to monitor the prevalence of physical activity assessment and promotion among children through integration of a standard pediatric physical activity vital sign in health care delivery. Estimated costs: medium.

Strategy 9: Expand the use of data from wearable devices for monitoring physical activity in at-risk patients. Estimated costs: medium.

Strategy 10: Conduct surveillance of cardiorespiratory fitness and muscle strength testing among at-risk populations in health care settings. Estimated costs: high.

Strategy 11: Ensure that national health care delivery surveys include questions about physical activity assessment and counseling in health care settings. Estimated costs: low.

Strategy 12: Conduct surveillance of physical activity levels in the health care workforce and related training programs. Estimated costs: high.

\section{Workplaces}

Strategy 13: Document existing surveillance efforts that capture physical activity, physical fitness, and sedentary behavior in the workplace and in employees' commutes to and from work, and identify opportunities to expand these efforts. Estimated costs: low.

Strategy 14: Convene public and private stakeholders to prioritize and implement consensus key measures to assess individual-level physical activity, physical fitness, and sedentary behavior surveillance in the workplace. Estimated costs: low.

Strategy 15: Develop consistent measures for physical activity in workplace designs and operations, policies, programs, culture, and climate, and use these measures in comprehensive surveillance of physical activity and physical fitness in the workplace. Estimated costs: high.

Strategy 16: Obtain longitudinal support and funding for the Workplace Health in America Survey. Estimated costs: medium.

\section{Community supports for physical activity}

Strategy 17: Prioritize a set of constructs and corresponding survey items to assess perception of community supports for active transportation and active recreation, incorporate the constructs and survey items into national surveillance systems, and promote their use at the local level. Estimated costs: low.

Strategy 18: Identify and compile geographic information system-based data sources and methods to facilitate national surveillance of community supports for physical activity. Estimated costs: low.

Strategy 19: Explore opportunities for partnering with professional organizations to query their membership about physical activity-supportive policies in the communities where they work and to share policy tracking data for surveillance purposes. Estimated costs: low.

Strategy 20: Develop and standardize methods for linking policies, self-reported surveillance systems, and environmental geospatial data to identify opportunities to support physical activity. Estimated costs: low.

Strategy 21: Identify a brief set of prioritized constructs and methods that could be assessed using audits (observations) of streets, parks, and other relevant public spaces. Estimated costs: high.

Strategy 22: Identify methods to assess physical activity events, programs, social environments, and promotion resources. Estimated costs: medium.

Note: Table is adapted from Pate, Sallis, and Pollack Porter, Surveillance of physical activity: Actions needed to support new federal guidelines. Am J Pub Health, 2020; 110(1):87-89. See the full report at nationalacademies.org/PhysicalActivitySurveillance for the accompanying implementation actions. Source-National Academies of Science, Engineering, and Medicine.

collecting data useful in both guiding the design of interventions and in evaluating the results of those interventions. Public health conceptualizations of physical activity interventions, such as the National Physical Activity Plan, ${ }^{28}$ are based on evidence that factors at multiple levels (eg, psychological, social, organizational, environmental, policy) influence physical activity and consensus that interventions are needed within multiple sectors of society (eg, health care, education, media, and transportation). Thus, the NASEM report ${ }^{24}$ consists mainly of proposals that would complement current surveillance by systematically assessing implementation of interventions and tracking trends in the documented influences on physical activity.

The NASEM report recommended actions to provide more subgroup-specific data on physical activity interventions and influences that would allow identification of subgroups at higher or lower risk, based on both demographics (eg, age, sex, race/ethnicity) and geography (eg, rural/suburban/urban and neighborhood). The proposed enhanced surveillance system would provide information on which interventions are being implemented, which precursors of behavior change are moving in favorable directions, 
and which subgroups are experiencing improvements. This information, along with physical activity data from the current systems, would provide detailed evidence about which types of interventions are most needed for each major population subgroup. These enhancements are needed because large majorities of American adults and adolescents are not fully meeting physical activity guidelines, and large disparities exist across population subgroups. ${ }^{1}$

\section{Information on Person-Level Physical Activity Behavior}

Most of the existing resources for physical activity surveillance in the United States are designed to monitor the prevalence of compliance with physical activity guidelines at the level of individual persons. Nonetheless, major gaps remain in surveillance of physical activity behavior in the US population, and actions should be taken to fill these gaps. Key actions include:

- Children under high school age: YRBS assesses self-reported physical activity behavior in high school students on a regular basis. But no existing system regularly monitors physical activity behavior in younger children. The Physical Activity Guidelines for Americans includes specific recommendations for children and youth, ages 3-18 years. ${ }^{13}$ Hence, additional physical activity surveillance activities are needed to regularly assess compliance with these guidelines across that entire age range.

- Types of physical activity behavior: No existing surveillance system regularly assesses the prevalence of participation in specific types of physical activity behavior. Partial assessments of specific behaviors are provided by the BRFSS, which asks respondents to report their 2 most frequent forms of physical activity; the YRBS, which assesses enrollment in physical education among high school students ${ }^{16}$; and the National Household Travel Survey, which reports on transportationrelated physical activity behaviors, such as walking and bicycling to destinations. ${ }^{21}$ But a comprehensive assessment of types of physical activity is needed to provide information that is critical in targeting public health interventions to promote physical activity.

- Settings and locations for physical activity: Knowledge of the physical and social settings in which people engage in physical activity is needed to design effective settings-based strategies for increasing physical activity in the population. No existing system monitors, in a comprehensive manner, the physical locations (eg, home, worksite, school, street, park, recreation center), or social environment (eg, organized group, single partner, individual) in which people typically participate in physical activity. Action should be taken to establish such a system.

- Device-based measurement of physical activity: Accelerometry provides for objective measurement of time spent in physical activity across a range of intensity levels and has been used in NHANES to assess compliance with physical activity guidelines across age groups from children to older adults. However, the procedures used in NHANES varied across measurement cycles, which has limited efforts to monitor change in objectively measured physical activity at the population level. We strongly encourage continued use of devices to measure physical activity, but comparability of measures across time should be ensured in future surveillance efforts.
- Prevalence estimates below the state level: Current resources for surveillance of physical activity lack granularity, and no large-scale system exists to provide prevalence estimates for compliance with physical activity guidelines at the county, municipal, or census tract levels. BRFSS and YRBS provide prevalence estimates at the state level, but many efforts to promote physical activity are planned and executed at the local level. National efforts should be supported by surveillance protocols that also operate at the local level.

\section{Information on Community Resources, Programs, and Policies}

The NASEM report calls for identifying actions to improve surveillance of the diverse set of policies, programs, initiatives, and resources that could be considered physical activity "interventions." A fundamental challenge is the diversity of the interventions themselves. Interventions designed to change people directly (ie, the individual level) include classes (exercise, dance, and physical education), counseling/coaching, communication campaigns, and incentives. Design of built environments that can affect physical activity applies across multiple scales, such as buildings, neighborhoods, cities/towns, and transportation systems, which cross jurisdictions. Policies are made by governments at all levels, several agencies within governments, as well as by public (schools) and private (businesses) organizations.

Key actions to enhance surveillance of physical activity "interventions" include:

- Community-based physical activity programs: Physical activity programs are available in most communities, and in many communities, a large and diverse set of physical activity programs are offered by numerous public and private organizations. These programs range from motor development programs for preschoolers, offered by faith-based organizations, to chair exercise programs for elders, provided by public recreation agencies. At present, there is no coordinated system, at any jurisdictional level, for monitoring availability and participation in these programs, and it is important to document inequities in program availability across communities. Because community-based programs are so numerous and diverse, it will be very challenging to develop a fully comprehensive, national system for monitoring availability and participation in such programs. The NASEM report recommends starting by developing a system for monitoring communitylevel availability of youth physical activity and sports programs, and then pilot testing that system in selected communities. This approach could and should be applied to programs serving other population subgroups, such as adult employees of private and public sector employers, people residing in socioeconomically disadvantaged communities, and retirees living independently or in congregate settings.

- Community resources for physical activity: Physical activity behavior is influenced by the physical environmental characteristics of communities. These include availability of and access to resources, such as parks, trails, sidewalks, green spaces, and supports for active transport, such as protected bike facilities and streets closed to automobile traffic. The Guide to Community Preventive Services ${ }^{29}$ recommends the combination of access to destinations, indicated by mixed land use, and transportation improvements that support safe and convenient walking, bicycling, and transit use. Some national databases include built environment variables, ${ }^{30}$ but data on 
key variables, such as sidewalks, bicycle facilities, health clubs, and parks, are either missing or of limited quality.

- Policies supporting physical activity: Some existing federal and state policies have almost certainly exerted powerful, positive influences on physical activity in the US population. These include state mandates for school physical education, Title IX of the federal Higher Education Act of 1972, which made sports programs widely available to girls and women, and the Americans with Disabilities Act, which expanded access to physical activity resources for persons with a wide range of disabilities. Federal, state, and local policies related to transportation and community planning can impact physical activity. Civil rights policies can affect access of historically disadvantaged groups to physical activity programs and resources. The NASEM report recommends establishment of a database for physical activity-related laws and policies, with an emphasis on state and local policies. ${ }^{24}$

- Local data: Few local data exist regarding community supports and physical activity interventions. The inequitable distribution of built environment features within cities and counties, such as presence and quality of parks, sidewalks, and protected bicycle facilities, ${ }^{31,32}$ demonstrates the need for surveillance at the local level. Because local data generally are not available, local public health departments do not have systematic data on which to base planning. Multiple local agencies have responsibility for parks, transportation, and land use infrastructure and policies. Though many of these agencies routinely collect data, they may not prioritize the specific variables of most interest to physical activity and public health professionals. Thus, public health partnerships with "nonhealth" agencies could be developed to support data collection activities that would be of mutual benefit, as recommended in the NASEM report. ${ }^{24}$ It is possible that technological approaches, such as data mining and crowd sourcing, could make local data collection feasible. ${ }^{33,34}$ Assistance likely to help local departments conduct surveillance includes providing, from the national level, low-cost, validated measures; software to enable scoring and analysis of results; and training and support of local epidemiologists. Local health departments could then share their data with a national agency so that prevalence, trends, and inequities could be compared across local jurisdictions.

\section{Call to Action: Cross-Sector Partnerships}

The US National Physical Activity Plan was created by a diverse coalition of organizations with the aim of fostering a national culture that supports physically active lifestyles. The Plan was originally released in 2010 and updated in $2016 .{ }^{28}$ It recommends a comprehensive set of policies, programs, and initiatives designed to increase physical activity in all segments of the US population. Although the Plan was designed to stimulate interventions to increase physical activity, lessons from the Plan are relevant to improving physical activity surveillance.

The Plan recognizes that though the goal is to improve health and quality of life, physical activity is primarily influenced by actions outside of public health and health care. The Plan is based on an assumption that meaningful increases in population physical activity in the United States cannot be achieved unless multiple sectors are engaged in changing environments, policies, and practices within their purview. Thus, the Plan is organized around nine societal sectors: Public Health; Healthcare; Education; Transportation, Land Use, and Community Planning; Parks, Recreation and Fitness; Business and Industry; Faith-based Settings; Mass Media; and Sport. ${ }^{28}$

There are interdisciplinary working groups for each sector, and these groups are building personal relationships and organizational partnerships to advance toward the Plan's goals. These relationships and partnerships could be applied to planning and implementing some of the physical activity surveillance actions recommended in the NASEM report. ${ }^{24}$

\section{Summary}

Physical inactivity is a prevalent and serious cause of public health burden in the United States. Although there has been long-term surveillance of physical activity of adults and high school students, many gaps exist in physical activity surveillance in the United States. The most significant gap is the lack of national surveillance of physical activity programs, policies, and environments that could guide intervention plans. A recent National Academies of Science, Engineering, and Medicine consensus report provided recommendations for creating a more comprehensive surveillance system that is expected to be useful in prioritizing and targeting interventions to increase population-level physical activity and reduce inequities in the United States. Improved surveillance will require partnerships among public health authorities and public and private collaborators representing diverse sectors.

\section{References}

1. Physical Activity Guidelines Advisory Committee. 2018 Physical Activity Guidelines Advisory Committee Scientific Report. Washington, DC: US DHHS; 2018.

2. Physical Activity Guidelines for Americans Midcourse Report Subcommittee, President's Council on Fitness Sports and Nutrition. Physical Activity Guidelines for Americans Midcourse Report: Strategies to Increase Physical Activity Among Youth. Washington, DC; 2012.

3. Powell KE, King AC, Buchner DM, et al. The scientific foundation for the physical activity guidelines for Americans, 2nd edition. J Phys Act Health. 2018;16(1):1-11. doi:10.1123/jpah.2018-0618

4. U.S. Department of Health and Human Services. Physical Activity Guidelines Advisory Committee Report. Washington, DC: USDHHS; 2008.

5. U.S. Department of Health and Human Services. Physical Activity and Health: A Report of the Surgeon General. Atlanta: USDHSS/ CDC; 1996.

6. DiPietro L, Buchner DM, Marquez DX, Pate RR, Pescatello LS, Whitt-Glover MC. New scientific basis for the 2018 U.S. Physical Activity Guidelines. J Sport Health Sci. 2019;8(3):197-200. PubMed ID: 31193291 doi:10.1016/j.jshs.2019.03.007

7. Jakicic JM, Powell KE, Campbell WW, et al. Physical activity and the prevention of weight gain in adults: a systematic review. Med Sci Sports Exerc. 2019;51(6):1262-1269. PubMed ID: 31095083 doi:10. 1249/MSS.0000000000001938

8. Pescatello LS, Buchner DM, Jakicic JM, et al. Physical activity to prevent and treat hypertension: A systematic review. Med Sci Sports Exerc. 2019;51(6):1314-1323. PubMed ID: 31095088 doi:10.1249/ MSS.0000000000001943

9. Pate RR, Hillman CH, Janz KF, et al. Physical activity and health in children younger than 6 years: a systematic review. Med Sci Sports 
Exerc. 2019;51(6):1282-1291. PubMed ID: 31095085 doi:10.1249/ MSS.0000000000001940

10. Department of Health. Australia's Physical Activity and Sedentary Behaviour Guidelines. Canberra, Australia: Australian government, Department of Health; 2019.

11. Chief Medical Officers of England Scotland Wales and Northern Ireland. Start Active, Stay Active: A Report on Physical Activity for Health from the Four Home Countries Chief Medical Officers. London: Department of Health, Physical Activity, Health Improvement and Protection; 2011.

12. U.S. Department of Health and Human Services. 2008 Physical Activity Guidelines for Americans. US Department of Health and Human Services. 2008. http://www.health.gov/paguidelines/. Accessed 2017 April 23.

13. U.S. Department of Health and Human Services. Physical Activity Guidelines for Americans, 2nd ed. 2018. https://health.gov/ paguidelines/second-edition/

14. Pickens CM, Pierannunzi C, Garvin W, Town M. Surveillance for certain health behaviors and conditions among states and selected local areas-behavioral risk factor surveillance system, United States, 2015. MMWR Surveill Summ. 2018;67(9):1-90. PubMed ID: 29953431 doi:10.15585/mmwr.ss6709a1

15. Centers for Disease Control and Prevention. Behavioral risk factor surveillance system. 2020. https://www.cdc.gov/brfss/index.html.

16. Centers for Disease Control and Prevention. Youth risk behavior surveillance system — questionnaires, 2019. 2019. https://www.cdc. gov/healthyyouth/data/yrbs/questionnaires.htm. Accessed February 27, 2020.

17. Centers for Disease Control and Prevention. National health and nutrition examination survey. 2020. https://www.cdc.gov/nchs/ nhanes/index.htm.

18. Centers for Disease Control and Prevention. National health interview survey. 2020. https://www.cdc.gov/nchs/nhis/index.htm.

19. Centers for Disease Control and Prevention. National youth fitness survey. 2013. https://www.cdc.gov/nchs/nnyfs/index.htm. Accessed February 27, 2018.

20. US Bureau of Labor Statistics. American time use survey. 2021. https://www.bls.gov/tus/.

21. Federal Highway Administration. National household travel survey. 2017. https://nhts.ornl.gov/.

22. U.S. Department of Health and Human Services, Centers for Disease Control and Prevention. Results from the School Health Policies and Practices Study 2016. Washington, DC: US DHHS; 2017.
23. National Cancer Institute. The Classification of Laws Associated with School Students (CLASS). Washington, DC: National Cancer Institute; 2018.

24. National Academies of Sciences Engineering, and Medicine. Implementing Strategies to Enhance Public Health Surveillance of Physical Activity in the United States. Washington, DC: The National Academics Press; 2019.

25. Fulton JE, Carlson SA, Ainsworth BE, et al. Strategic Priorities for Physical Activity Surveillance in the United States. Med Sci Sports Exerc. 2016;48(10):2057-2069. PubMed ID: 27187094 doi:10.1249/ MSS.0000000000000989

26. Pate RR, Berrigan D, Buchner D, et al. Actions to Improve Physical Activity Surveillance in the United States. Washington, DC: National Academy of Medicine; 2018.

27. Pate RR, Sallis JF, Pollack Porter KM. Surveillance of physical activity: actions needed to support new federal guidelines. Am J Public Health. 2020;110(1):87-89. PubMed ID: 31800284 doi:10. 2105/AJPH.2019.305443

28. National Physical Activity Plan Alliance. The U.S. national physical activity plan. 2016. http://physicalactivityplanorg/docs/2016NPAP_ Finalforwebsitepdf

29. Guide to Community Preventive Services. Combined built environment features help communities get active. 2018. https://www. thecommunityguide.org/content/combined-built-environment-featureshelp-communities-get-active. Accessed March 4, 2021.

30. Environmental Protection Agency. Smart location mapping. 2020; https://www.epa.gov/smartgrowth/smart-location-mapping. Accessed March 4, 2021.

31. Engelberg JK, Conway TL, Geremia C, et al. Socioeconomic and race/ethnic disparities in observed park quality. BMC Public Health. 2016;16(1):395. PubMed ID: 27176854 doi:10.1186/s12889-0163055-4

32. Thornton CM, Conway TL, Cain KL, et al. Disparities in pedestrian streetscape environments by income and race/ethnicity. SSM Popul Health. 2016;2:206-216. PubMed ID: 27314057 doi:10.1016/j. ssmph.2016.03.004

33. Brabham DC, Ribisl KM, Kirchner TR, Bernhardt JM. Crowdsourcing applications for public health. Am J Prev Med. 2014;46(2):179_ 187. PubMed ID: 24439353 doi:10.1016/j.amepre.2013.10.016

34. Kamel Boulos MN, Resch B, Crowley DN, et al. Crowdsourcing, citizen sensing and sensor web technologies for public and environmental health surveillance and crisis management: trends, OGC standards and application examples. Int J Health Geogr. 2011;10(1):67. PubMed ID: 22188675 doi:10.1186/1476-072X-10-67 